\title{
A Game-Theoretic Model of Cholera with Optimal Personal Protection Strategies
}

\author{
Julia Kobe $^{1} \cdot$ Neil Pritchard $^{2} \cdot$ Ziaqueria Short $^{3} \cdot$ Igor V. Erovenko $^{2}$. \\ Jan Rychtáŕ ${ }^{2}$. Jonathan T. Rowell ${ }^{2}$
}

Received: 30 December 2016 / Accepted: 23 July 2018 / Published online: 10 September 2018

(c) Society for Mathematical Biology 2018

\begin{abstract}
Cholera is an acute gastro-intestinal infection that affects millions of people throughout the world each year, primarily but not exclusively in developing countries. Because of its public health ramifications, considerable mathematical attention has been paid to the disease. Here we consider one neglected aspect of combating cholera: personal participation in anti-cholera interventions. We construct a game-theoretic model of cholera in which individuals choose whether to participate in either vaccination or clean water consumption programs under assumed costs. We find that relying upon individual compliance significantly lowers the incidence of the disease as long as the cost of intervention is sufficiently low, but does not eliminate it. The relative costs of the measures determined whether a population preferentially adopts a single preventative measure or employs the measure with the strongest early adoption.
\end{abstract}

Keywords Cholera $\cdot$ Game theory $\cdot$ ESS $\cdot$ Personal protection $\cdot$ Vaccination

\section{Introduction}

Cholera is an acute infectious disease of the small intestine marked by significant morbidity and mortality (Barua 1992), including a 20\% mortality rate for children under the age of 5 years (Bryce et al. 2005). The disease causes severe diarrhea in patients over a period of 3-7 days (Miller Neilan et al. 2010; Wang and Modnak 2011;

Igor V. Erovenko

igor@uncg.edu

1 Department of Applied Mathematics, Wentworth Institute of Technology, Boston, MA 02115, USA

2 Department of Mathematics and Statistics, University of North Carolina at Greensboro, Greensboro, NC 27402, USA

3 Department of Biological Sciences, Winston-Salem State University, Winston-Salem, NC 27110, USA 
Jensen et al. 2006), which may be accompanied by vomiting, loss of skin elasticity, thirst, muscle cramps (Zuckerman et al. 2007), and, in extreme cases, fatal dehydration within a matter of hours (WHO 2016). The waterborne bacterium Vibrio cholerae has been identified as the causative agent of the disease (Miller Neilan et al. 2010; Faruque and Nair 2008; Colwell and Huq 1994; WHO 2016). Human infection results from a fecal-oral mode of transmission, most often via the consumption of contaminated food or water (WHO 2010a; Tauxe et al. 1995). The majority of these cholera cases arise in areas without reliable access to clean water and proper sanitation, such as in areas affected by poverty, poor environmental conditions, or other humanitarian crises (WHO 2016; Franco et al. 1997).

Cholera was once confined to the Ganges Delta and neighboring territories (Peters and Wendt 1885; Colwell 1996; Barua 1992; Wachsmuth et al. 1994), but since escaping its natural reservoir at the start of the nineteenth century, the disease has spread globally through a series of seven pandemics (Epstein 1993; Miller Neilan et al. 2010; Amako et al. 1987; Colwell et al. 2003). In 2001 alone, 58 countries struggled with outbreaks of cholera (Colwell et al. 2003). Annually there are 2.4-2.8 million cases reported worldwide (Colwell et al. 2003), although some estimates range from 1.44.3 million cases, with a death rate of 28,000-142,000 per annum (Ali et al. 2012). There are also concerns that the incidence of the disease is still underreported. The current pandemic began in Indonesia in 1961 (Codeço 2001) before spreading around the world in the 1960s and 1970s from Africa and the Mediterranean to Japan (WHO 2016; Faruque and Nair 2008). In 1991, cholera returned to parts of Latin America where it had been absent for a century (WHO 2000). Recent epidemics in Zimbabwe in 2008-2009 (WHO 2016, 2009; Mukandavire et al. 2011a) and Haiti in 2010 (WHO 2010b; Bertuzzo et al. 2011; Mukandavire et al. 2013) resulted in major loss of life, and outbreaks continue to emerge worldwide (Gil et al. 2004; Seas et al. 2000; Bertuzzo et al. 2008).

There are over 200 known serotypes of $V$. cholerae based upon the somatic $\mathrm{O}$ antigen (Ruiz-Moreno et al. 2010), yet only two serotypes, O1 and O139, are linked to the cholera disease (Lipp et al. 2002). The classical O1 strain was responsible for the first six pandemics, while the more recent El Tor strain has been prominent during the seventh (Kaper et al. 1995). The bacteria, or vibrios, produce an enterotoxin that acts on the mucosal epithelium of the human host's small intestine (Mukandavire et al. 2011b). During shedding, V. cholerae enters a brief hyperinfective state (700 times the normal rate) (Sanches et al. 2011; Merrell et al. 2002), and even asymptomatic carriers can spread the disease with disastrous consequences, as can ship ballast which is suspected in the case of Peru (WHO 2016; Bertuzzo et al. 2011; Piarroux et al. 2011; Seas et al. 2000).

Both direct and indirect modes of transmission contribute to the spread of cholera (Shuai et al. 2012; Tien and Earn 2010; Rosenberg 1987). Controls have been implemented to combat the disease at these different transmission points through public education, vaccination, and therapeutic activities (Mwasa and Tchuenche 2011; Bertuzzo et al. 2011). In developing countries where poor water quality and sanitation are common, the utilization of alternative water sources, e.g., bottled water, reduces the incidence of imbibing contaminated water (WHO 2016), while improvised filtration has proven a relatively inexpensive but effective alternative approach to the 
same problem (Colwell et al. 2003). Additionally, there are two approved classes of vaccines: Dukerol, a monvalent vaccine that also protects against E. coli; and Shanchol/mORCVAX, two related bivalent vaccines that do not provide this secondary protection (WHO 2016; Gaffga et al. 2007; WHO 2010a). Both types of vaccines are administered orally through two doses and have been used in several successful vaccination campaigns (Calain et al. 2004; Chaignat et al. 2008; WHO 2016) (however, see (Cyranoski 2011) for a discussion of disagreements). Both classes offer a strong immunity to cholera (66\% for Shanchol/mORCVAX, $84 \%$ for Dukerol) that last between two to three years (Levine et al. 1981).

Many theoretic efforts have been applied to the study of the dynamics of cholera epidemics (Capasso and Paveri-Fontana 1979; Pascual et al. 2002; Tien and Earn 2010; Mukandavire et al. 2011b; Wang and Liao 2012; Wang and Modnak 2011), with Codeço (2001) model serving as the foundation for most subsequent studies. Model extensions have incorporated variable reservoir volume (Pascual et al. 2002), hyperinfectivity during shedding (Hartley et al. 2006; Shuai et al. 2012), direct person to person transmission (Pascual et al. 2006), partial or temporary immunity (Sanches et al. 2011; Safi et al. 2013; Shuai et al. 2012), spatial spread (Bertuzzo et al. 2008, 2009, 2011), and co-infection with other diseases (Okosun and Makinde 2014; Mushayabasa and Bhunu 2012). Miller Neilan et al. (2010) investigated the efficacy of intervention protocols conducted under the auspices of state or health organization campaigns for post-infection therapy, vaccination, and sanitation.

The application of game theory to the efficacy of disease prevention is fairly recent (Tuite et al. 2011; Shim et al. 2012) despite its established role in modeling other biological phenomena (Hofbauer and Sigmund 1998; Maynard Smith 1982; Mesterton-Gibbons 2000; Vincent and Brown 2012; Broom and Rychtář 2013). Many preventative or therapeutic actions are informed not by mandatory external controls (Miller Neilan et al. 2010) but rather by individual decisions to participate that balance the perceived costs of the action (financial, associated health risks) against the treatment's promised effectiveness. Vaccination games have already addressed smallpox (Bauch et al. 2003), influenza (Galvani et al. 2007), measles (Shim et al. 2012), rubella (Shim et al. 2009), and toxoplasmosis (Sykes and Rychtár 2015). This general framework of personal decisions has also been applied to other methods including deploying insecticide-treated cattle (Crawford et al. 2015), mosquito repellent (Dorsett et al. 2015), and insecticide-treated bed nets (Broom et al. 2016).

Here we construct a game-theoretic model of individual decisions concerning two interventions - vaccination and clean water usage - in an environment at risk for a cholera outbreak. These treatments may be undertaken singly or in conjunction with each other. Beginning with an epidemiological SVIRB compartment model under the assumption that immunity is temporary (Mwasa and Tchuenche 2011), we allow the vaccination rate parameter and clean water consumption rate to reflect the compliance of the population and then test the optimal individual response to the group's behavior. First we calculate both the disease-free and endemic states of the model and compute the disease's basic reproductive number. The payoff differential for using or declining a single dose of vaccine generates an optimal vaccination strategy. Similarly, we determine the optimal strategy for the consumption of clean water. While neither voluntary vaccination nor clean water usage is sufficient to eradicate cholera, the adoption of 
optimal personal strategies does push the dynamic parameters close to levels necessary for herd immunity for sufficiently small intervention costs. Additionally when the protocols are considered together, their relative costs establish which protective measure is more likely to be adopted by the population.

\section{Methods}

We will use a slightly simplified version of the cholera model with public health interventions presented by Mwasa and Tchuenche (2011). The human population is divided into four groups: those who are susceptible to the disease $(S)$, those presently vaccinated $(V)$, individuals currently infected $(I)$, and recovered individuals $(R)$. The concentration of $V$. colerae bacteria in the aquatic environment is denoted by $B$.

Disease-free individuals enter the population at a constant rate $\Lambda$ either by birth or immigration, and there is a natural per capita mortality $\mu_{1}$. Letting $r \in[0,1]$ denote the portion of an individual's daily water consumption deriving from clean sources, the rate of ingestion of contaminated water is presumed equal to

$$
\beta(r)=\beta_{0}(1-r)
$$

where $\beta_{0}$ is the total daily ingestion rate. Infection of susceptibles is then governed by the Michaelis-Menten rate $\beta(r) B /(K+B)$, with half-saturation term $K$.

The susceptible individuals are vaccinated at a rate $\varphi$. Individuals receive complete immunity from the disease while they stay in the vaccinated class; however, protection wears off at a rate $\omega$ returning individuals to the susceptible class. Infected individuals either succumb to cholera infection (represented by an increase in mortality, $d$ ) or enter the recovered class at a rate $\alpha$. Recovered individuals are immune for the remainder of their lives. Infected individuals shed bacteria into the aquatic environment at a rate $e$. Bacteria themselves undergo decay at a rate $\mu_{2}$. A schematic diagram of our model is presented in Fig. 1.

The flow chart in Fig. 1 yields the following system of differential equations:

$$
\begin{aligned}
\frac{\mathrm{d} S}{\mathrm{~d} t} & =\Lambda+\omega V-\left(\mu_{1}+\varphi+\beta(r) \frac{B}{K+B}\right) S, \\
\frac{\mathrm{d} V}{\mathrm{~d} t} & =\varphi S-\left(\mu_{1}+\omega\right) V, \\
\frac{\mathrm{d} I}{\mathrm{~d} t} & =\beta(r) \frac{B}{K+B} S-\left(\mu_{1}+d+\alpha\right) I, \\
\frac{\mathrm{d} R}{\mathrm{~d} t} & =\alpha I-\mu_{1} R, \\
\frac{\mathrm{d} B}{\mathrm{~d} t} & =e I-\mu_{2} B .
\end{aligned}
$$

Table 1 contains the values of the parameters used in our model. We assume the individuals' life expectancy of 63.5 years, and the recruitment rate of new individuals 


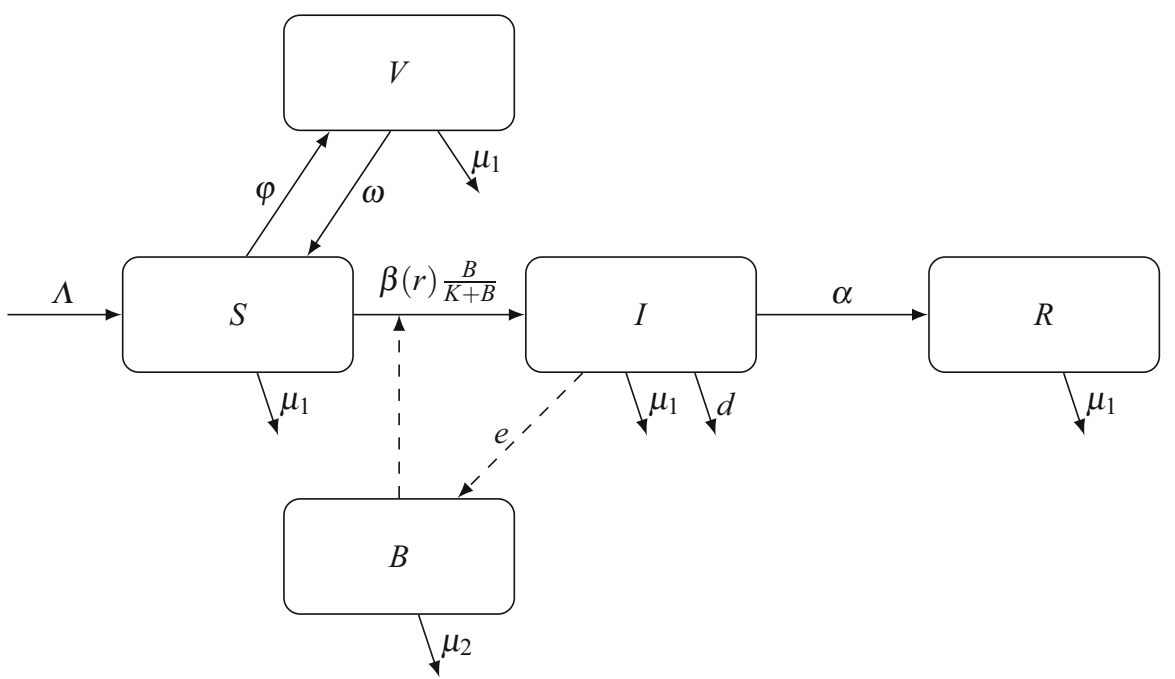

Fig. 1 Compartment model of cholera with vaccination and clean water usage as personal protection strategies

Table 1 Summary of the parameters of the model

\begin{tabular}{|c|c|c|c|}
\hline Symbol & Meaning & Value & Source \\
\hline$\Lambda$ & Recruitment rate of humans & 23 days $^{-1}$ & Assumed \\
\hline$\alpha$ & Recovery rate & .2 days $^{-1}$ & Hartley et al. (2006) \\
\hline$\beta_{0}$ & $\begin{array}{l}\text { Ingestion rate of } V . \text { cholerae bacteria } \\
\text { by humans }\end{array}$ & .2134 days $^{-1}$ & Tuite et al. (2011) \\
\hline$\mu_{1}$ & Natural human death rate & $(63.5 \cdot 365)^{-1}$ days $^{-1}$ & Assumed \\
\hline$\mu_{2}$ & Decay rate of bacteria & .33 days $^{-1}$ & Felsenfeld (1963) \\
\hline$\omega$ & Rate of vaccine wear off & $(2 \cdot 365)^{-1}$ days $^{-1}$ & WHO (2014) \\
\hline$\varphi$ & Vaccination rate & Varies & \\
\hline$d$ & Disease induced death rate & .015 days $^{-1}$ & Barzilay et al. (2013) \\
\hline$e$ & $\begin{array}{l}\text { Rate of bacteria production by an } \\
\text { infected individual }\end{array}$ & 10 cells $/ \mathrm{ml} \mathrm{day}^{-1}$ & Codeço (2001) \\
\hline$K$ & Half-saturation constant & $10^{6}$ cells $/ \mathrm{ml}$ & Codeço (2001) \\
\hline$C^{v}$ & Relative cost of vaccination & Varies & \\
\hline$C^{w}$ & $\begin{array}{l}\text { Relative cost of complete protection } \\
\text { through clean water }\end{array}$ & Varies & \\
\hline$r$ & Proportion of clean water used & Varies & \\
\hline
\end{tabular}

$\Lambda$ is 23 days $^{-1}$. We chose our value of $\Lambda$ so that the resulting population is sufficiently, but not unrealistically, large at equilibrium. 
The disease model admits two potential equilibria, one with the disease present and one without it. The disease-free equilibrium (DFE) of our model is given by

$$
\left(S^{0}, V^{0}, I^{0}, R^{0}, B^{0}\right)=\left(\frac{\Lambda\left(\mu_{1}+\omega\right)}{\mu_{1}\left(\mu_{1}+\omega+\varphi\right)}, \frac{\Lambda \varphi}{\mu_{1}\left(\mu_{1}+\omega+\varphi\right)}, 0,0,0\right) .
$$

The basic reproduction number $\mathscr{R}_{0}$ represents the number of secondary cases which one infected individual would produce in a completely susceptible population [i.e., the DFE (3)]. Using the next-generation matrix method of van den Driessche and Watmough (2002) to compute the basic reproduction number for our model, we consider the dynamics of the infectious classes $I$ and $B$ in isolation of the rest of system (2). Let $F$ be the sensitivity matrix of the appearance rate of new incidents of infection to $I$ and $B$, and $V$ the sensitivity matrix in shifts between infection states, then

$$
F=\left[\begin{array}{cc}
0 & \frac{\beta_{0}(1-r) \Lambda\left(\mu_{1}+\omega\right)}{\mu_{1} K\left(\mu_{1}+\omega+\varphi\right)} \\
e & 0
\end{array}\right] \text { and } V=\left[\begin{array}{cc}
\mu_{1}+d+\alpha & 0 \\
0 & \mu_{2}
\end{array}\right]
$$

The reproduction number is equal to the spectral radius of the matrix $F V^{-1}$ :

$$
\mathscr{R}_{0}=\sqrt{\frac{e \beta_{0}(1-r) \Lambda\left(\mu_{1}+\omega\right)}{\mu_{1} \mu_{2} K\left(\mu_{1}+\omega+\varphi\right)\left(\mu_{1}+d+\alpha\right)}} .
$$

When $\mathscr{R}_{0}>1$, the disease can invade, and the system converges to the endemic equilibrium (EE)

$$
\begin{aligned}
S^{*} & =\frac{\mu_{2}\left(\mu_{1}+d+\alpha\right)\left(K+B^{*}\right)}{e \beta_{0}(1-r)}, \\
V^{*} & =\frac{\mu_{2} \varphi\left(\mu_{1}+d+\alpha\right)\left(K+B^{*}\right)}{e \beta_{0}(1-r)\left(\mu_{1}+\omega\right)}, \\
I^{*} & =\frac{\mu_{2} B^{*}}{e}, \\
R^{*} & =\frac{\mu_{2} \alpha B^{*}}{e \mu_{1}}, \\
B^{*} & =\frac{\left(\mathscr{R}_{0}^{2}-1\right) \mu_{1} K\left(\mu_{1}+\omega+\varphi\right)}{\mu_{1}\left(\mu_{1}+\omega+\varphi\right)+\beta_{0}(1-r)\left(\mu_{1}+\omega\right)} .
\end{aligned}
$$

Note that $\mathscr{R}_{0}-1$ must be positive for these expressions to be biologically reasonable.

\section{Results}

We initially assume that only one type of personal protection strategy, either vaccination or clean water usage, is available at a time, and compute the optimal strategies in each scenario. We then combine our findings of optimal vaccination rate and clean 
water usage to investigate what happens when both personal protection strategies for cholera are available.

\subsection{Vaccination as a Personal Protection Strategy}

First consider vaccination as the sole personal protection strategy available against cholera. The clean water consumption rate is thus set at $r=0$ in our epidemiological model (2). The threshold value of the vaccination rate necessary to achieve herd immunity is obtained by setting the basic reproduction number in (5) equal to $\mathscr{R}_{0}=1$ and solving for $\varphi$. We find that the threshold vaccination rate is

$$
\varphi_{\mathrm{HI}}=\frac{e \beta_{0} \Lambda\left(\mu_{1}+\omega\right)}{\mu_{1} \mu_{2} K\left(\mu_{1}+d+\alpha\right)}-\left(\mu_{1}+\omega\right) .
$$

The graph of $\mathscr{R}_{0}$ as a function of the vaccination rate $\varphi$ is shown in Fig. 2a. For values of $\varphi$ less than $\varphi_{\mathrm{HI}}$, the disease remains endemic, while for values of $\varphi$ greater than $\varphi_{\mathrm{HI}}$ the disease is eradicated.

Next, we construct and solve the game-theoretic model of optimal individual vaccination strategies. An individual chooses to either vaccinate or not vaccinate, indexed $v$ and $n v$, respectively. To evaluate these two strategies, we assign an expected payoff to each strategy following the general framework of Bauch and Earn (2004):

$$
\begin{aligned}
E_{v} & =-C_{v}-\pi_{v} C_{i}, \\
E_{n v} & =-\pi_{n v} C_{i} .
\end{aligned}
$$

The expected payoffs of individuals who choose to vaccinate or not vaccinate, respectively, are functions of the probabilities of getting infected $\pi_{v}$ and $\pi_{n v}$, for a vaccinated or non-vaccinated individual, respectively, and the costs of the vaccination and getting infected $C_{v}$ and $C_{i}$, respectively. These costs include both direct costs, such as the financial cost of the vaccine or of medical treatment, and indirect costs, such as potential side effects of the vaccine or malady or morbidity risks from infection.

Since scaling payoff functions by a constant do not change the outcome of the game, we divide both equations by $C_{i}$ to obtain

$$
\begin{aligned}
E_{v} & =-C^{v}-\pi_{v}, \\
E_{n v} & =-\pi_{n v},
\end{aligned}
$$

where $C^{v}=C_{v} / C_{i}$ is the cost of vaccination relative to the cost of infection.

Within our model (2), individuals in the background could receive multiple vaccinations over the course of their life, alternating between the susceptible and vaccinated classes. Here we consider a focal individual who will decide whether or not to vaccinate within a set period of time (typically shorter than $1 / \varphi$ ) based upon a short-term risk calculation. Hence the perceived probability of getting infected for a non-vaccinating 

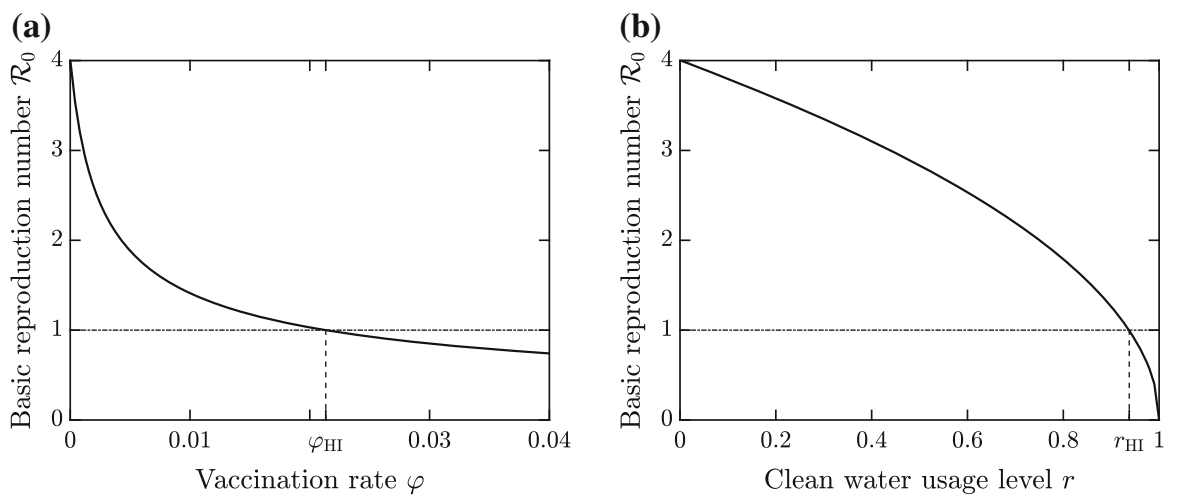

Fig. 2 The basic reproduction number $\mathscr{R}_{0}$ as a function of the vaccination rate $\varphi$; the threshold value of the vaccination rate necessary to reach herd immunity is denoted by $\varphi_{\mathrm{HI}}$, and $\mathbf{b}$ a function of the proportion of clean water used by the population $r$; the threshold value of the clean water usage necessary to reach herd immunity is denoted by $r_{\mathrm{HI}}$

individual is

$$
\pi_{n v}=\frac{\beta_{0} B /(K+B)}{\beta_{0} B /(K+B)+\mu_{1}}=\frac{\beta_{0} B}{\beta_{0} B+\mu_{1}(K+B)} .
$$

Similarly, the perceived probability of a singularly vaccinated individual becomes infected is

$$
\pi_{v}=\frac{\omega}{\mu_{1}+\omega} \frac{\beta_{0} B}{\beta_{0} B+\mu_{1}(K+B)} .
$$

This short-term calculation for a focal individual is a reasonable proxy for the weak selection pressures driving the population-level vaccination rate $\varphi$, particularly when adoption rates are small. One of the reasons we do not model a lifetime vaccination strategy of a focal individual is because the conditions influencing vaccination may change over time, including vaccine efficacy, monetary or social costs, and any potential side effects. The short-term calculation reflects the natural cognitive limitation of individuals to account for all possibilities. Moreover in our model, individuals are not affected by their prior vaccination history, and all associated costs are additive, so the decision-making event is reasonably limited to the immediate instance. If the population is under-vaccinated, focal individuals will be incentivized to adopt the treatment, driving the population rate upward. Likewise, focal individuals forgo treatment when the population is over-vaccinated. This approach would be less appropriate with more frequent or continuous decisions (e.g., use of clean water).

Let $\varphi_{\text {pop }}$ be the vaccination rate of the entire population. If $\varphi_{\text {pop }}>\varphi_{\mathrm{HI}}$, then $\mathscr{R}_{0}<1$ and the population reaches the disease-free equilibrium (3). In this case, $B=B^{0}=0$ and the probability of getting infected is zero: $\pi_{v}=\pi_{n v}=0$. So any given focal individual has no incentive to vaccinate and may in fact be favored to forgo vaccination if there are any perceived costs associated with it. If instead $\varphi_{\text {pop }}<\varphi_{\mathrm{HI}}$, then $\mathscr{R}_{0}>1$ 
and the population reaches the endemic equilibrium (6). The focal individual considers the difference between the short-term expected payoffs of the two strategies:

$$
\Delta E=E_{v}-E_{n v}=-C^{v}+\frac{\mu_{1}}{\mu_{1}+\omega} \frac{\beta_{0} B}{\beta_{0} B+\mu_{1}(K+B)} .
$$

The focal individual should vaccinate when $\Delta E>0$ and not vaccinate when $\Delta E<0$. When the disease is endemic we have $B=B^{*}$, and hence an individual should vaccinate when

$$
C^{v}<\frac{\mu_{1}}{\mu_{1}+\omega} \frac{\beta_{0} B^{*}}{\beta_{0} B^{*}+\mu_{1}\left(K+B^{*}\right)}
$$

and not vaccinate when

$$
C^{v}>\frac{\mu_{1}}{\mu_{1}+\omega} \frac{\beta_{0} B^{*}}{\beta_{0} B^{*}+\mu_{1}\left(K+B^{*}\right)} .
$$

If the disease is endemic, the optimal vaccination rate $\varphi_{\mathrm{NE}}$ at which it does not matter whether a given individual chooses to vaccinate or not solves the equation

$$
C^{v}=\frac{\mu_{1}}{\mu_{1}+\omega} \frac{\beta_{0} B^{*}}{\beta_{0} B^{*}+\mu_{1}\left(K+B^{*}\right)} .
$$

Substituting the expression for $B^{*}$ in (6) (while keeping in mind that we set $r=0$ when vaccination is the sole personal protection strategy available) and solving for $\varphi$, we obtain

$$
\varphi_{\mathrm{NE}}=x \frac{z e \beta_{0} \Lambda-y \mu_{1} K\left(z-x\left(\mu_{1}+\beta_{0}\right) C^{v}\right)}{y \mu_{1} K\left(z+x \mu_{1} C^{v}\right)}
$$

where

$$
\begin{aligned}
& x=\mu_{1}+\omega, \\
& y=\mu_{2}\left(\mu_{1}+d+\alpha\right), \\
& z=x\left(\beta_{0}-C^{v}\left(\mu_{1}+\beta_{0}\right)\right)-\beta_{0} \omega .
\end{aligned}
$$

The graph of the optimal vaccination rate $\varphi_{\mathrm{NE}}$ as a function of the relative cost of vaccination $C^{v}$ when the disease is endemic is shown in Fig. 3a. Observe that $\varphi_{\mathrm{NE}}$ never exceeds the herd immunity threshold $\varphi_{\mathrm{HI}}$, and they are equal only when the cost of vaccination is zero. Also, $\varphi_{\mathrm{NE}}=0$ as long as the relative cost of vaccination is greater than a threshold value $C_{\max }^{v}$.

\subsection{Clean Water Usage as a Personal Protection Strategy}

Now we consider clean water usage as a personal protection strategy against cholera. For this we set $\varphi=\omega=0$ in the epidemiological model we constructed in the 
(a)

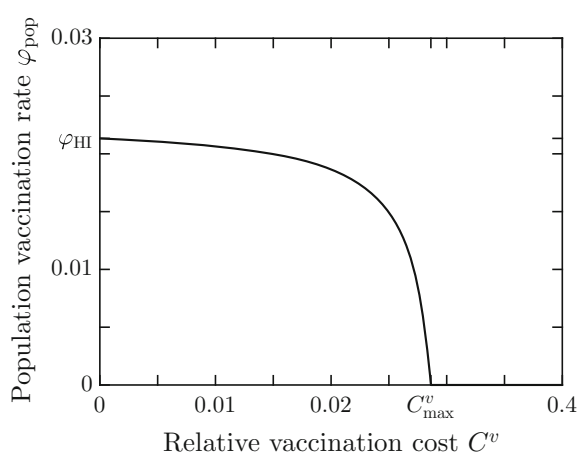

(b)

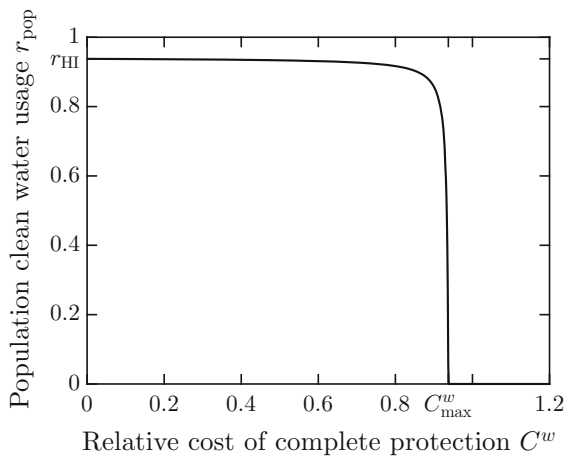

Fig. 3 a The graph of the optimal vaccination rate $\varphi_{\mathrm{NE}}$ as a function of the relative cost of vaccination $C^{v}$. In the parameter area below the graph, an individual should vaccinate, and in the parameter area above the graph, an individual should not vaccinate. Nobody should vaccinate when the cost of vaccination relative to the cost of the disease is greater than a threshold value $C_{\max }^{v}$. b The graph of the optimal level of clean water usage $r_{\mathrm{NE}}$ as a function of the relative cost of complete protection $C^{w}$. In the parameter area below the graph, an individual should use clean water only, and in the parameter area above the graph, an individual should not use any clean water. Nobody should use any clean water when the cost of complete protection relative to the cost of the disease is greater than a threshold value $C_{\max }^{w}$

previous section. By setting the basic reproduction number in (5) equal to 1 , we find the threshold value of the clean water usage level in the population necessary to reach herd immunity:

$$
r_{\mathrm{HI}}=1-\frac{\mu_{1} \mu_{2}\left(\mu_{1}+d+\alpha\right) K}{e \beta_{0} \Lambda} .
$$

The graph of $\mathscr{R}_{0}$ as a function of the proportion of clean water used by the population is shown in Fig. 2b. For values of $r$ less than $r_{\mathrm{HI}}$ the, disease remains endemic, and for values greater than $r_{\mathrm{HI}}$, the disease is eradicated.

An individual's strategy is the proportion $r$ of clean water (either bottled or filtered water) he/she uses as part of his/her daily water consumption. When the population is in endemic equilibrium, the probability that a focal individual gets infected is $\lambda /\left(\lambda+\mu_{1}\right)$, where $\lambda=\beta(r) B^{*} /\left(K+B^{*}\right)$ is the force of infection. The force of infection depends on (a) the rate of ingestion of contaminated water by a focal individual, determined by the factor $\beta(r)$, which is equal to $\beta_{0}(1-r)$ by (1); and (b) the concentration of bacteria in contaminated water $B^{*}$ which, in turn, depends on the clean water usage level in the population $r_{\text {pop }}$ given the expression for $B^{*}$ in (6). We thus have

$$
\lambda=\lambda\left(r, r_{\text {pop }}\right)=\beta_{0}(1-r) \frac{B^{*}}{K+B^{*}} .
$$


Again adopting the framework of Bauch and Earn (2004), we express the payoff of a focal individual using strategy $r$ in the population using strategy $r_{\text {pop }}$ as

$$
\begin{aligned}
E\left(r, r_{\text {pop }}\right) & =-\frac{\lambda\left(r, r_{\text {pop }}\right)}{\lambda\left(r, r_{\text {pop }}\right)+\mu_{1}}-C^{w} r \\
& =-\frac{\beta_{0}(1-r) B^{*}}{\beta_{0}(1-r) B^{*}+\mu_{1}\left(K+B^{*}\right)}-C^{w} r,
\end{aligned}
$$

where $C^{w}=C_{w} / C_{i}$ is the cost of complete $(r=1)$ protection $C_{w}$ relative to the cost of contracting cholera $C_{i}$ for the individual. These costs include both direct costs, such as the cost of clean water or the cost of medical treatment in case of infection, and indirect costs, such as morbidity risks of the cholera infection.

To optimize an individual's response to the current environment, we note that

$$
\begin{aligned}
\frac{\partial E\left(r, r_{\text {pop }}\right)}{\partial r} & =\frac{\mu_{1} \beta_{0} B^{*}\left(K+B^{*}\right)}{\left(\beta_{0}(1-r) B^{*}+\mu_{1}\left(K+B^{*}\right)\right)^{2}}-C^{w}, \\
\frac{\partial^{2} E\left(r, r_{\text {pop }}\right)}{\partial r^{2}} & =\frac{2 \mu_{1} \beta_{0}^{2}\left(B^{*}\right)^{2}\left(K+B^{*}\right)}{\left(\beta_{0}(1-r) B^{*}+\mu_{1}\left(K+B^{*}\right)\right)^{3}}>0 .
\end{aligned}
$$

It follows that $E\left(r, r_{\text {pop }}\right)$ is a convex function of $r$, and so its globally maximum value is attained at either 0 or 1 . The corresponding payoffs are

$$
E\left(0, r_{\text {pop }}\right)=-\frac{\beta_{0} B^{*}}{\beta_{0} B^{*}+\mu_{1}\left(K+B^{*}\right)},
$$

where the focal individual does not use any clean water at all, but faces the risk of contracting cholera, and

$$
E\left(1, r_{\text {pop }}\right)=-C^{w}
$$

where the focal individual uses clean water exclusively, and consequently is guaranteed to not catch cholera while incurring the full cost of complete protection relative to the cost of the infection.

The best strategy for a focal individual depends on $B^{*}$, which is a function of the personal protection strategy used by the population $r_{\text {pop }}$, and the relative cost of complete protection $C^{w}$. If

$$
\frac{\beta_{0} B^{*}}{\beta_{0} B^{*}+\mu_{1}\left(K+B^{*}\right)}<C^{w},
$$

then the risk of infection is lower than the relative cost of protection and the individual should not use any clean water. If

$$
\frac{\beta_{0} B^{*}}{\beta_{0} B^{*}+\mu_{1}\left(K+B^{*}\right)}>C^{w},
$$


then the risk of infection is higher than the relative cost of protection and the individual should use clean water only to avoid contracting cholera.

The optimal strategy $r_{\mathrm{NE}}$ at which it does not matter whether one adopts clean water usage or not is the solution to the equation

$$
E\left(0, r_{\mathrm{NE}}\right)=E\left(1, r_{\mathrm{NE}}\right)
$$

Using (27) and (28), we can rewrite this equation as

$$
\frac{\beta_{0} B^{*}}{\beta_{0} B^{*}+\mu_{1}\left(K+B^{*}\right)}=C^{w} .
$$

Substituting the expression for $B^{*}$ in (6) (while keeping in mind that we set $\varphi=\omega=0$ when clean water usage is the only personal protection strategy available) and solving for $r$, we obtain

$$
r_{\mathrm{NE}}=\frac{y \mu_{1} K-u e \Lambda}{y \mu_{1} K C^{w}-u e \Lambda}
$$

where

$$
u=\beta_{0}-C^{w}\left(\mu_{1}+\beta_{0}\right)
$$

and $y$ is given by (20). The graph of the optimal clean water usage level $r_{\mathrm{NE}}$ as a function of the relative cost of complete protection $C^{w}$ is shown in Fig. 3b. Observe that $r_{\mathrm{NE}}$ never exceeds the herd immunity threshold $r_{\mathrm{HI}}$, and they are equal only when the cost of complete protection is zero. Also, $r_{\mathrm{NE}}=0$ as long as the relative cost of complete protection is greater than a threshold value $C_{\max }^{w}$.

The behavior of the two graphs shown in Fig. 3 is consistent with previous results. Bauch and Earn (2004) observe that increasing the value of $\mathscr{R}_{0}$ results in a sharper dropoff in the graph of the optimal strategy as a function of the relative cost of protection. In the limit of very large values of $\mathscr{R}_{0}$, the graph approaches a step function. In our model, the basic reproduction number value for water-only model is usually larger than the one for vaccination-only model for endemic case (Fig. 2). Hence the graph of the optimal level of clean water usage experiences a steeper decline once the relative cost of the protective measure approaches the threshold value.

\subsection{Combining Vaccination and Clean Water Usage to Combat Cholera}

Finally, we consider the case where both vaccination and clean water usage are available as personal protection strategies against cholera. We already know the optimal vaccination rate $\varphi_{\mathrm{NE}}$ when no clean water is used from (18), and the optimal clean water usage $r_{\mathrm{NE}}$ when no-one vaccinates from (33). When both personal protection strategies are available, the optimal vaccination rate becomes a function of clean water usage level, $\varphi_{\mathrm{NE}}(r)$. Likewise, the optimal clean water usage becomes a function of 
the vaccination rate, $r_{\mathrm{NE}}(\varphi)$. Substituting the expression for $B^{*}$ in (6) into (17) and (32) and solving for $\varphi$ and $r$, respectively, we obtain

$$
\varphi_{\mathrm{NE}}(r)=x \frac{z(r) e \beta_{0}(1-r) \Lambda-y \mu_{1} K\left(z(r)-x\left(\mu_{1}+\beta_{0}(1-r)\right) C^{v}\right)}{y \mu_{1} K\left(z(r)+x \mu_{1} C^{v}\right)}
$$

and

$$
r_{\mathrm{NE}}(\varphi)=\frac{y K\left(x \mu_{1} C^{w}+v(\varphi)\left(1-C^{w}\right)\right)-x u е \Lambda}{x y \mu_{1} K C^{w}-x u e \Lambda}
$$

where

$$
\begin{aligned}
z(r) & =x\left(\beta_{0}(1-r)-C^{v}\left(\mu_{1}+\beta_{0}(1-r)\right)\right)-\beta_{0}(1-r) \omega \\
v(\varphi) & =\mu_{1}(\varphi+x)
\end{aligned}
$$

$x$ is given by (19), $y$ is given by (20), and $u$ is given by (34). Figure 4 shows the results of combining vaccination and clean water usage protocols.

One strategy may dominate the other, or either strategy may be employed, with the particular one chosen determined by early adopters in the population. This is naturally determined by the corresponding relative costs: a strategy with lower relative cost (with respect to the maximum threshold cost for the corresponding strategy) is more likely to be adopted than a strategy with higher relative cost. To determine for what combinations of relative costs of vaccination $C^{v}$ and clean water usage $C^{w}$ one strategy is dominant or when either may be adopted exclusively, we let $r_{0}$ be the clean water usage level such that $\varphi_{\mathrm{NE}}\left(r_{0}\right)=0$ and $\varphi_{0}$ be the vaccination rate such that $r_{\mathrm{NE}}\left(\varphi_{0}\right)=0$. Numerical simulations demonstrate that the case when both $r_{0}>r_{\mathrm{NE}}$ and $\varphi_{0}>\varphi_{\mathrm{NE}}$ is impossible for the parameter values listed in Table 1. Consequently, there are three primary cases to consider:

- if $r_{0}>r_{\mathrm{NE}}$ and $\varphi_{0}<\varphi_{\mathrm{NE}}$, then the black line (optimal vaccination rate) stays above the gray line (optimal level of clean water usage), hence vaccination is the dominant strategy (cf. Fig. 4d);

- if $\varphi_{0}>\varphi_{\mathrm{NE}}$ and $r_{0}<r_{\mathrm{NE}}$, then the gray line stays above the black line, hence clean water usage is the dominant strategy (cf. Fig. 4c); and

- if $r_{0}<r_{\mathrm{NE}}$ and $\varphi_{0}<\varphi_{\mathrm{NE}}$, then the two lines intersect, hence either strategy may be adopted exclusively by the population (cf. Fig. 4e, f).

The parameter space regions in Fig. $4 \mathrm{~b}$ corresponding to each these scenarios are computed as follows. Setting Eqs. (35) and (36) equal to zero and solving for $r$ and $\varphi$, respectively, we obtain

$$
r_{0}=1-\mu_{1} \frac{x e \Lambda C^{v}+y \mu_{1} K}{e \beta_{0} \Lambda\left(\mu_{1}-x C^{v}\right)} \quad \text { and } \quad \varphi_{0}=x \frac{u e \Lambda-y \mu_{1} K}{y \mu_{1} K\left(1-C^{w}\right)} .
$$

Vaccination is the dominant strategy (black region) when $r_{0}>r_{\mathrm{NE}}$ :

$$
1-\mu_{1} \frac{x e \Lambda C^{v}+y \mu_{1} K}{e \beta_{0} \Lambda\left(\mu_{1}-x C^{v}\right)}>\frac{y \mu_{1} K-u e \Lambda}{y \mu_{1} K C^{w}-u e \Lambda},
$$


(a)

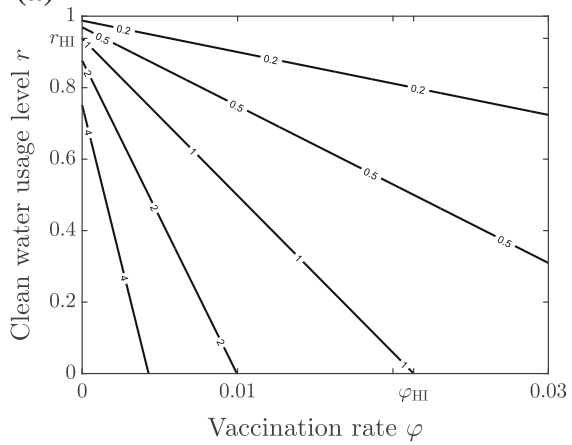

(c)

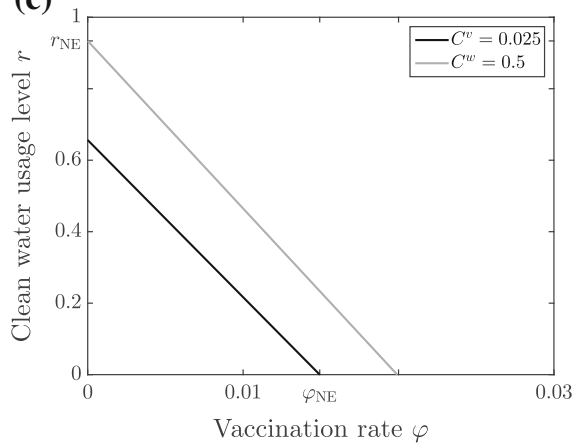

(e)

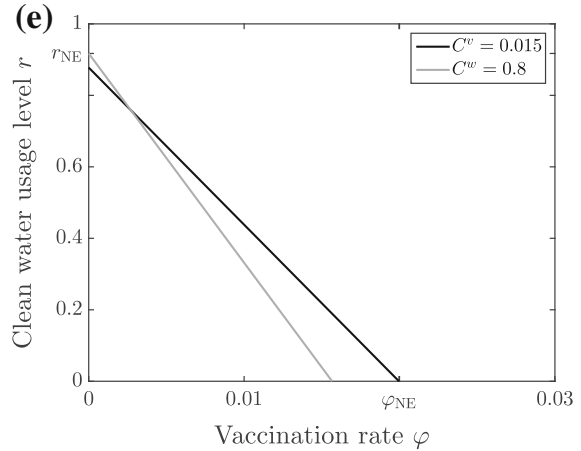

(b)

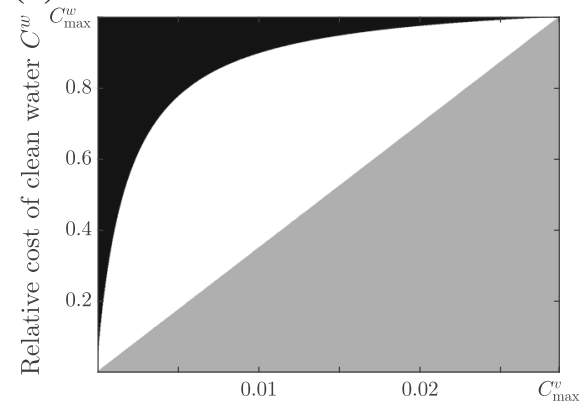

Relative vaccination cost $C^{v}$

(d)
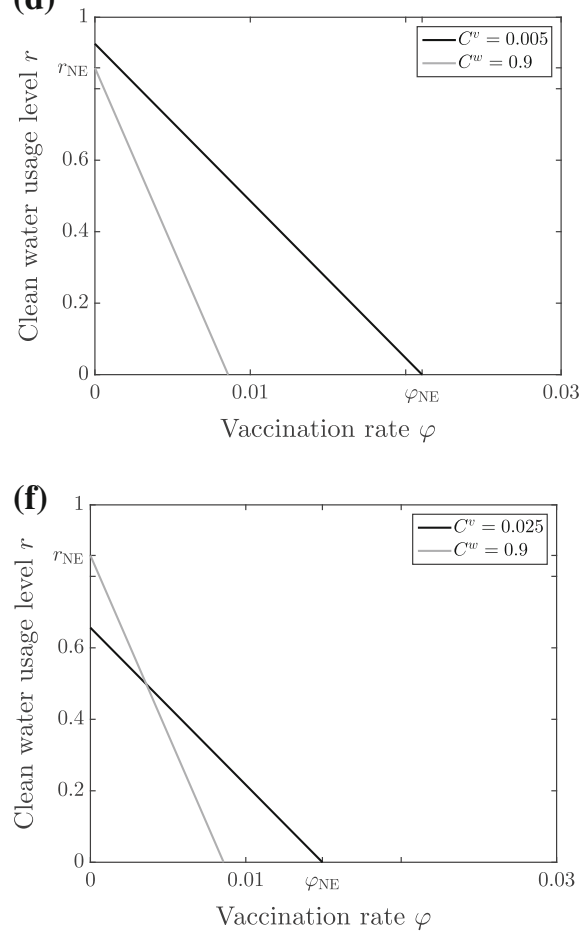

Fig. 4 Combining vaccination and clean water usage strategies. a Contour plot of $\mathscr{R}_{0}$ as a function of both vaccination rate $\varphi$ and clean water usage level $r$. b Regions in the $\left(C^{v}, C^{w}\right)$-parameter space in which one personal strategy is used exclusively or in which both are used. Color codes: black - vaccination only, gray — clean water only, white — either strategy may be used exclusively. The graphs $\mathbf{c}-\mathbf{f}$ compare optimal vaccination rates (black lines) to optimal clean water usage (gray lines) when both strategies are employed. c Clean water usage is the dominant strategy. $\mathbf{d}$ Vaccination is the dominant strategy. e-f Either strategy may be used exclusively 
and clean water usage is the dominant strategy (gray region) when $\varphi_{0}>\varphi_{\mathrm{NE}}$ :

$$
\frac{u e \Lambda-y \mu_{1} K}{y \mu_{1} K\left(1-C^{w}\right)}>\frac{z e \beta_{0} \Lambda-y \mu_{1} K\left(z-x\left(\mu_{1}+\beta_{0}\right) C^{v}\right)}{y \mu_{1} K\left(z+x \mu_{1} C^{v}\right)} .
$$

Otherwise, either preventative measure is a possible action, but early adoption will determine which measure is used exclusively by a given population (white region). The cut-off values of the relative costs in the graph in Fig. $4 \mathrm{~b}$ are the threshold values $C_{\max }^{v}$ and $C_{\max }^{w}$ beyond which no-one vaccinates (cf. Fig. 3a) or uses any clean water (cf. Fig. 3b), respectively. This phenomenon is illustrated in Fig. 4b as follows. At the upper boundary of the graph, where the relative cost of clean water usage approaches $C_{\max }^{w}$, vaccination is the dominant strategy since no-one will use the costly clean water protection. Similarly, at the right boundary of the graph, where the relative cost of vaccination approaches $C_{\max }^{v}$, clean water usage is the dominant strategy since no-one will vaccinate. If both relative costs $C^{v}$ and $C^{w}$ exceed their corresponding thresholds, then neither protective strategy is going to be used by the population since their benefits do not compensate the high cost (the corresponding region in Fig. 4b not shown, but occurs above and to the right of the plot window).

\section{Discussion}

Following the general framework of Bauch and Earn (2004), we adopted a cholera epidemiology model with different public health interventions (Mwasa and Tchuenche 2011) and constructed a game-theoretic study of voluntary participation in two intervention measures designed to reduce the incidence of cholera: vaccination and clean water consumption. Voluntary participation in these policies presents a subtle challenge for disease management: if a sufficient proportion of the population is already participating, then even slight costs or risks associated with the intervention can outweigh the expected risk posed by infection. As a result, individual self-interest might preclude complete eradication of a preventable disease even when costs incurred are very low (Geoffard and Philipson 1997). Although complete eradication of cholera was not possible through voluntary compliance, the optimal personal strategies for vaccination or clean water usage did reach levels close to those required for herd immunity, and cholera can be reduced to low endemic levels provided the relative costs of these interventions are sufficiently low. On the other hand, if the cost of intervention relative to the potential cost of contracting the disease exceed a certain threshold value, then the corresponding intervention becomes non-viable as individuals would rather face the risk of infection than take costly protective measures.

When both vaccination and clean water consumption are available to the population, the relative costs of these two measures determine which strategy (or a combination of both) is more likely to be adopted by the population. If one strategy has low relative cost (with respect to the corresponding maximum threshold cost) while the other strategy has high relative cost, then individuals will choose the lower cost alternative exclusively. A combination of both strategies could be employed by the population only when the corresponding relative costs are commensurate and there is appeal for 
the adoption of a novel treatment when another measure has achieved its equilibrium usage rate. In our particular model, such an outcome is not observed. Instead of co-adoption, one method will characterize the population based upon early adopters, but neither method is dominant over the other. It is important to note that there is disagreement over parameter values in the literature (Chao et al. 2011; Grad et al. 2012; Akman et al. 2016). It remains an open question as to how robust the set of potential outcomes are to variations in these parameter values, and other parameters (e.g., for other diseases) could promote multiple-treatment equilibria rather than mutually exclusive ones. Future validation of the model system may require the use of genetic-algorithms to better estimate these values (Akman and Schaefer 2015).

In this form of analysis, individuals possess perfect knowledge of the direct and indirect costs of accepting available intervention measures or contracting cholera, as well as the participation levels adopted by the rest of the population. Individual evaluations of the respective outcomes, however, may differ from a simple utilitarian perspective (Bauch and Earn 2004). Socially-driven opinions, economic hardships, cultural norms, public education and advocacy campaigns can all adjust parametric values assigned within the model, resulting in a decision game that, to some extent, deviates from an "objective" game. For example, vaccination scares can dramatically reduce the coverage rate because widespread safety concerns heighten the perceived personal risks from accepting the vaccination.

As a case in point, Colwell et al. (2003) introduced the use of sari cloth as an improvised filtration system to several Bangladeshi villages between September 1999 and July 2002. These villages traditionally used unfiltered water where cholera was present (Briscoe 1978) and a lack of suitable fuel prevented the boiling of the water (Colwell et al. 2003). Cultural norms against polluting one's self also hampered use of vaccines (Colwell and Huq 1994; Briscoe 1978). By folding sari cloth 4-8 times, however, it was possible to create a mesh that removed particles as small as $20 \mu \mathrm{m}$. This method of reducing the consumption of contaminated water was both economically inexpensive and raised no cultural objections. Consequently, use of sari cloth had an exceptionally high acceptance rate, and participating villages experienced a $48 \%$ reduction in cholera cases.

The ecology of $V$. cholerae and disease progression point to further developments of this paper. Our model presupposed that cholera could only persist in the presence of a human host population; however, vibrios are autochthonous in many coastal aquatic communities (Colwell et al. 1977, 1981; Colwell and Huq 1994) where there is a reservoir in which they reside in a commensal association with zooplankton (Colwell and Huq 1994; Kaneko and Colwell 1973). Endemic settings typically have one or two cholera peaks during the year (Faruque et al. 1998) that are timed to when a zooplankton bloom crashes and disintegrating shells release vibrios directly into the sea water (Gooday 1990; Flach et al. 1992). This can be represented in the equation of bacterial concentration through a zooplankton-mediated growth term.

Additionally, it may take up to 3 weeks to develop immunity after vaccination (Jertborn et al. 1993), necessitating the inclusion of a time delay component. Moreover, acquired immunity to cholera, whether by vaccine or recovery after exposure, is only temporary (Levine et al. 1981) and may only be partially effective in preventing transmission (Safi et al. 2013). Further mild exposure may lead to a shorter immunity period 
(King et al. 2008). These factors require additional model pathways from vaccinated to infected classes, or from recovered to susceptible and infected classes.

Finally as with the static analysis of Bauch and Earn (2004), there is an assumption that the system has reached equilibrium before individuals optimize their personal adoption strategies. Achieving this equilibrium state, however, may occur on a timescale comparable to, or longer than, the one on which personal strategies change. The probability of getting infected thus changes dynamically along with disease prevalence. At the beginning of the epidemic, when the force of infection is high, the probability of getting infected is higher, and hence individuals have more incentive to vaccinate than at later stages of the epidemiological dynamics when the disease prevalence approaches stable equilibrium values. Consequently, the next model iteration should therefore include the rate of vaccination and the use of clean water as state variables.

Acknowledgements This research was conducted as part of a Research Experiences for Undergraduates program held at the University of North Carolina at Greensboro in summer 2016 and was funded by the National Science Foundation Grant DMS-1359187, awarded to JR and JTR as PI and co-PI, respectively. The authors also thank E. Hollifield and M. Leshowitz, who served as graduate assistants during the 2016 REU program.

\section{References}

Akman O, Schaefer E (2015) An evolutionary computing approach for parameter estimation investigation of a model for cholera. J Biol Dyn 9(1):147-158

Akman O, Corby M, Schaefer E (2016) Examination of models for cholera: insights into model comparison methods. Lett Biomath 3(1):93-118

Ali M, Lopez A, You Y, Kim Y, Sah B, Maskery B, Clemens J (2012) The global burden of cholera. Bull World Health Organ 90:209-218A

Amako K, Shimodori S, Imoto T, Miake S, Umeda A (1987) Effects of chitin and its soluble derivatives on survival of Vibrio cholerae O1 at low temperature. Appl Environ Microbiol 53(3):603-605

Barua D (1992) History of cholera. In: Barua D, Greenough WB III (eds) Cholera. Springer, Berlin, pp $1-36$

Barzilay E, Schaad N, Magloire R, Mung K, Boncy J, Dahourou G, Mintz E, Steenland M, Vertefeuille J, Tappero J (2013) Cholera surveillance during the Haiti epidemic: the first 2 years. N Engl J Med 368(7):599-609

Bauch C, Earn D (2004) Vaccination and the theory of games. Proc Nat Acad Sci USA 101(36):13,39113,394

Bauch C, Galvani A, Earn D (2003) Group interest versus self-interest in smallpox vaccination policy. Proc Nat Acad Sci USA 100(18):10,564-10,567

Bertuzzo E, Azaele S, Maritan A, Gatto M, Rodriguez-Iturbe I, Rinaldo A (2008) On the space-time evolution of a cholera epidemic. Water Res Res 44(1):W01424

Bertuzzo E, Casagrandi R, Gatto M, Rodriguez-Iturbe I, Rinaldo A (2009) On spatially explicit models of cholera epidemics. J R Soc Interface 7:321-333

Bertuzzo E, Mari L, Righetto L, Gatto M, Casagrandi R, Blokesch M, Rodriguez-Iturbe I, Rinaldo A (2011) Prediction of the spatial evolution and effects of control measures for the unfolding Haiti cholera outbreak. Geophys Res Lett 38(6):L06403

Briscoe J (1978) The role of water supply in improving health in poor countries (with special reference to Bangladesh). Am J Clin Nutr 31(11):2100-2113

Broom M, Rychtár J (2013) Game-theoretical models in biology. Chapman and Hall/CRC, Boca Raton

Broom M, Rychtář J, Spears-Gill T (2016) The game-theoretical model of using insecticide-treated bed-nets to fight malaria. Appl Math 7:852-860 
Bryce J, Boschi-Pinto C, Shibuya K, Black R et al, Group WCHER (2005) WHO estimates of the causes of death in children. Lancet 365(9465):1147-1152

Calain P, Chaine JP, Johnson E, Hawley ML, O'Leary MJ, Oshitani H, Chaignat CL (2004) Can oral cholera vaccination play a role in controlling a cholera outbreak? Vaccine 22(19):2444-2451

Capasso V, Paveri-Fontana S (1979) A mathematical model for the 1973 cholera epidemic in the European Mediterranean region. Rev Epidemiol Sante Publique 27(2):121-132

Chaignat CL, Monti V, Soepardi J, Petersen G, Sorensen E, Narain J, Kieny M (2008) Cholera in disasters: Do vaccines prompt new hopes? Expert Rev Vaccines 7(4):431-435

Chao D, Halloran M, Longini I (2011) Vaccination strategies for epidemic cholera in Haiti with implications for the developing world. Proc Nat Acad Sci USA 108(17):7081-7085

Codeço C (2001) Endemic and epidemic dynamics of cholera: the role of the aquatic reservoir. BMC Infect Dis $1: 1$

Colwell R (1996) Global climate and infectious disease: the cholera paradigm. Science 274(5295):2025

Colwell R, Huq A (1994) Environmental reservoir of Vibrio cholerae. The causative agent of cholera. Ann NY Acad Sci 740(1):44-54

Colwell R, Kaper J, Joseph S (1977) Vibrio cholerae, Vibrio parahaemolyticus, and other vibrios: occurrence and distribution in Chesapeake Bay. Science 198(4315):394-396

Colwell R, Seidler R, Kaper J, Joseph S, Garges S, Lockman H, Maneval D, Bradford H, Roberts N, Remmers E et al (1981) Occurrence of Vibrio cholerae serotype O1 in Maryland and Louisiana estuaries. Appl Environ Microbiol 41(2):555-558

Colwell R, Huq A, Islam M, Aziz K, Yunus M, Khan N, Mahmud A, Sack R, Nair G, Chakraborty J et al (2003) Reduction of cholera in Bangladeshi villages by simple filtration. Proc Nat Acad Sci USA 100(3):1051-1055

Crawford K, Lancaster A, Oh H, Rychtár J (2015) A voluntary use of insecticide-treated cattle can eliminate African sleeping sickness. Lett Biomath 2(1):91-101

Cyranoski D (2011) Cholera vaccine plan splits experts. Nature 469:273-274

Dorsett C, Oh H, Paulemond M, Rychtář J (2015) Optimal repellent usage to combat dengue fever. Bull Math Biol 78(5):916-922

Epstein P (1993) Algal blooms in the spread and persistence of cholera. Biosystems 31(2-3):209-221

Faruque S, Nair G (2008) Vibrio cholerae: genomics and molecular biology. Horizon Scientific Press, Poole

Faruque S, Albert M, Mekalanos J (1998) Epidemiology, genetics, and ecology of toxigenic Vibrio cholerae. Microbiol Mol Biol Rev 62(4):1301-1314

Felsenfeld O (1963) Some observations on the cholera (El Tor) epidemic in 1961-62. Bull World Health Organ 28(3):289-296

Flach J, Pilet P, Jolles P (1992) What's new in chitinase research? Experientia 48(8):701-716

Franco A, Fix A, Prada A, Paredes E, Palomino J, Johnson J, McCarter R, Guerra H, Morris J et al (1997) Cholera in Lima, Peru, correlates with prior isolation of Vibrio cholerae from the environment. Am J Epidemiol 146(12):1067-1075

Gaffga N, Tauxe R, Mintz E (2007) Cholera: A new homeland in Africa? Am J Trop Med Hyg 77(4):705-713

Galvani A, Reluga T, Chapman G (2007) Long-standing influenza vaccination policy is in accord with individual self-interest but not with the utilitarian optimum. Proc Nat Acad Sci USA 104(13):56925697

Geoffard PY, Philipson T (1997) Disease eradication: private versus public vaccination. Am Econ Rev 87(1):222-230

Gil A, Louis V, Rivera I, Lipp E, Huq A, Lanata C, Taylor D, Russek-Cohen E, Choopun N, Sack R et al (2004) Occurrence and distribution of Vibrio cholerae in the coastal environment of Peru. Environ Microbiol 6(7):699-706

Gooday G (1990) The ecology of chitin degradation. Adv Microb Ecol 11:387-430

Grad Y, Miller J, Lipsitch M (2012) Cholera modeling: challenges to quantitative analysis and predicting the impact of interventions. Epidemiology 23(4):523

Hartley D, Morris J Jr, Smith D (2006) Hyperinfectivity: A critical element in the ability of V. cholerae to cause epidemics? PLoS Med 3(1):e7

Hofbauer J, Sigmund K (1998) Evolutionary games and population dynamics. Cambridge University Press, Cambridge

Jensen M, Faruque S, Mekalanos J, Levin B (2006) Modeling the role of bacteriophage in the control of cholera outbreaks. Proc Nat Acad Sci USA 103(12):4652-4657 
Jertborn M, Svennerholm A, Holmgren J (1993) Evaluation of different immunization schedules for oral cholera B subunit-whole cell vaccine in Swedish volunteers. Vaccine 11(10):1007-1012

Kaneko T, Colwell R (1973) Ecology of Vibrio parahaemolyticus in Chesapeake Bay. J Bacteriol 113(1):2432

Kaper J, Morris J Jr, Levine M (1995) Cholera. Clin Microbiol Rev 8(1):48-86

King A, Ionides E, Pascual M, Bouma M (2008) Inapparent infections and cholera dynamics. Nature 454(7206):877-880

Levine M, Black R, Clements M, Cisneros L, Nalin D, Young C (1981) Duration of infection-derived immunity to cholera. J Infect Dis 143(6):818-820

Lipp E, Huq A, Colwell R (2002) Effects of global climate on infectious disease: the cholera model. Clin Microbiol Rev 15(4):757-770

Maynard Smith J (1982) Evolution and the theory of games. Cambridge University Press, Cambridge

Merrell D, Butler S, Qadri F, Dolganov N, Alam A, Cohen M, Calderwood S, Schoolnik G, Camilli A (2002) Host-induced epidemic spread of the cholera bacterium. Nature 417(6889):642-645

Mesterton-Gibbons M (2000) An introduction to game-theoretic modelling. American Mathematical Society, New York

Miller Neilan R, Schaefer E, Gaff H, Fister K, Lenhart S (2010) Modeling optimal intervention strategies for cholera. Bull Math Biol 72(8):2004-2018

Mukandavire Z, Liao S, Wang J, Gaff H, Smith D, Morris J (2011a) Estimating the reproductive numbers for the 2008-2009 cholera outbreaks in Zimbabwe. Proc Nat Acad Sci USA 108(21):8767-8772

Mukandavire Z, Tripathi A, Chiyaka C, Musuka G, Nyabadza F, Mwambi H (2011b) Modelling and analysis of the intrinsic dynamics of cholera. Differ Equ Dyn Syst 19(3):253-265

Mukandavire Z, Smith D, Morris J Jr (2013) Cholera in Haiti: reproductive numbers and vaccination coverage estimates. Sci Rep 3:997

Mushayabasa S, Bhunu C (2012) Is HIV infection associated with an increased risk for cholera? Insights from a mathematical model. Biosystems 109(2):203-213

Mwasa A, Tchuenche J (2011) Mathematical analysis of a cholera model with public health interventions. Biosystems 105(3):190-200

Okosun K, Makinde O (2014) A co-infection model of malaria and cholera diseases with optimal control. Math Biosci 258:19-32

Pascual M, Bouma M, Dobson A (2002) Cholera and climate: revisiting the quantitative evidence. Microbes Infect 4(2):237-245

Pascual M, Koelle K, Dobson A (2006) Hyperinfectivity in cholera: A new mechanism for an old epidemiological model? PLoS Med 3(6):e280

Peters J, Wendt E (1885) A treatise on Asiatic cholera. William Wood and Company, West Chester

Piarroux R, Barrais R, Faucher B, Haus R, Piarroux M, Gaudart J, Magloire R, Raoult D (2011) Understanding the cholera epidemic, Haiti. Emerg Infect Dis 17(7):1161-1168

Rosenberg C (1987) The cholera years: the United States in 1832, 1849, and 1866. University of Chicago Press, Chicago

Ruiz-Moreno D, Pascual M, Emch M, Yunus M (2010) Spatial clustering in the spatio-temporal dynamics of endemic cholera. BMC Infect Dis 10:51

Safi M, Melesse D, Gumel A (2013) Dynamics analysis of a multi-strain cholera model with an imperfect vaccine. Bull Math Biol 75(7):1104-1137

Sanches R, Ferreira C, Kraenkel R (2011) The role of immunity and seasonality in cholera epidemics. Bull Math Biol 73(12):2916-2931

Seas C, Miranda J, Gil A, Leon-Barua R, Patz J, Huq A, Colwell R, Sack R (2000) New insights on the emergence of cholera in Latin America during 1991: the Peruvian experience. Am J Trop Med Hyg 62(4):513-517

Shim E, Kochin B, Galvani A (2009) Insights from epidemiological game theory into gender-specific vaccination against rubella. Math Biosci Eng 6(4):839-854

Shim E, Grefenstette J, Albert S, Cakourus B, Burke D (2012) A game dynamic model for vaccine skeptics and vaccine believers: measles as an example. J Theor Biol 295:194-203

Shuai Z, Tien J, Van den Driessche P (2012) Cholera models with hyperinfectivity and temporary immunity. Bull Math Biol 74(10):2423-2445

Sykes D, Rychtář J (2015) A game-theoretic approach to valuating toxoplasmosis vaccination strategies. Theor Popul Biol 105:33-38 
Tauxe R, Mintz E, Quick R (1995) Epidemic cholera in the new world: translating field epidemiology into new prevention strategies. Emerg Infect Dis 1(4):141

Tien J, Earn D (2010) Multiple transmission pathways and disease dynamics in a waterborne pathogen model. Bull Math Biol 72(6):1506-1533

Tuite A, Tien J, Eisenberg M, Earn D, Ma J, Fisman D (2011) Cholera epidemic in Haiti, 2010: using a transmission model to explain spatial spread of disease and identify optimal control interventions. Ann Intern Med 154(9):593-601

van den Driessche P, Watmough J (2002) Reproduction numbers and sub-threshold endemic equilibria for compartmental models of disease transmission. Math Biosci 180(1-2):29-48

Vincent T, Brown J (2012) Evolutionary game theory, natural selection, and Darwinian dynamics. Cambridge University Press, Cambridge

Wachsmuth I, Blake P, Olsvik O (1994) Vibrio cholerae and cholera: molecular to global perspectives. ASM Press, New York

Wang J, Liao S (2012) A generalized cholera model and epidemic-endemic analysis. J Biol Dyn 6(2):568589

Wang J, Modnak C (2011) Modeling cholera dynamics with controls. Can Appl Math Q 19(3):255-273

Wkly Epidemiol Rec (2000) Cholera, 1999. 75(31):249-256

WHO (2009) Disease outbreak news: cholera in Zimbabwe-update 4

WHO (2010a) Cholera vaccines: WHO position paper. Wkly Epidemiol Rec 85(13):117-128

WHO (2010b) Disease outbreak news: cholera in Haiti—update 4

WHO (2014) Use of oral cholera vaccine in humanitarian emergencies

WHO (2016) Cholera (Fact sheet 107)

Zuckerman J, Rombo L, Fisch A (2007) The true burden and risk of cholera: implications for prevention and control. Lancet Infect Dis 7(8):521-530 